\section{SELECT4: A BASIC program for applying definitions of fairness in selection procedures}

\author{
BRIAN MULLEN \\ Syracuse University, Syracuse, $N Y$
}

SELECT4 is a BASIC program that allows the user to examine the results of applying four different statistical definitions of fairness to bivariate selection data involving two subgroups. Previously published programs (SELECT1 and SELECT2, Mullen, 1984; SELECT3, Mullen, 1985) illustrate basic bivariate selection principles. SELECT4 complements these programs by demonstrating whether one subgroup (the "minority" subgroup) is being treated fairly relative to another subgroup (the "majority" subgroup) (Hunter \& Schmidt, 1976; Hunter, Schmidt, \& Rauschenberger, 1977; Ledvinka, 1979).

Statistical definitions of fairness hold that "Fair is when both subgroups are treated the same in terms of (blank)." The various definitions of fairness differ in how the blank is filled in. Each definition of fairness relies upon a particular decision equivalent to define the attribute in terms of which the two subgroups should be treated the same. A decision equivalent is a ratio involving true positives, false positives, true negatives, and false negatives, resulting from the use of a given selection procedure. For each of four definitions of fairness, SELECT4 determines the extent to which the current selection procedure is fair, and determines the consequences of making the selection procedure fair according to each definition of faimess. The techniques implemented by this program could be useful to academics teaching industrial/organizational psychology courses as well as to practitioners in applied settings.

Input. After being prompted to enter the number of majority subgroup applicants and the number of minority subgroup applicants, the user is prompted to enter a predictor score and a criterion score for each of the applicants. The user is also prompted to enter a criterion cutoff or a minimal acceptable job performance score. Then the user is allowed to select from a menu the particular definition of fairness he/she would like to see implemented: Regression; Constant Ratio; Conditional Probability; or Culture Free (see references cited above).

Output. After the user inputs predictor scores, criterion scores, and the criterion cutoff, SELECT4 outputs some basic descriptive statistics ( $\bar{X}, \bar{Y}, S D_{X}, S D_{Y}$ ) and selection statistics $(r$, the least-squares regression formula $[Y=a$ $+b X]$, and the predictor cutoff associated with the entered criterion cutoff). These preliminary statistics are displayed for the majority subgroup data, for the minority subgroup data, and for the total group data (majority and minority combined).

The author's mailing address is Department of Psychology, Syracuse University, Syracuse, NY 13210.
After the user selects a particular definition of fairness from the menu, the program displays a verbal description of that definition, a characterization of that definition of fairness in terms of the corresponding decision equivalents. Then the program displays the current decision equivalent for the majority, the current decision equivalent for the minority, the new predictor cutoff for the minority that would be needed to make the selection procedure fair according to this definition of fairness, the resultant new decision equivalent for the minority, and the criterion score associated with this new predictor cutoff for the minority. The difference between this criterion score, associated with the new predictor cutoff for the minority, and the previously entered criterion cutoff illustrates the consequences of applying that particular definition of fairness with that particular data analysis. At this point, the user is allowed either to select an alternative definition of fairness or to end execution of the program.

Limitations. The program is designed to operate within the context of a positive relationship between predictor and criterion (i.e., a selection procedure with a positive validity coefficient). A selection procedure that uses a negative validity coefficient should be rescaled to represent a positive validity coefficient before entering the program. The program is designed to handle a maximum of 40 majority subgroup applicants, $\mathbf{4 0}$ minority subgroup applicants, and a total of 80 applicants. Depending upon the memory capacity of the user's system, these limitations can be extended indefinitely by altering the dimensions of the vectors defined in lines 120 and 130.

Language. The program is written in an extremely generic dialect of BASIC that should run on most BASIC systems with little or no modification. The program consists of 266 lines and takes up approximately 10800 bytes of RAM.

Availability. The program listing can be obtained free of charge by writing to the author.

\section{REFERENCES}

Hunter, J. E., \& SCHMIDT, F. L. (1976). Critical analysis of the statistical and ethical implications of various definitions of test bias. Psychological Bulletin, 85, 1053-1071.

Hunter, J. E., Schmidt, F. L., \& Rauschenberger, J. M. (1977). Faimess of psychological tests: Implications of four definitions for selection utility and minority hiring. Journal of Applied Psychology, 62, 245-260.

LeDVINKA, J. (1979). The statistical definition of fairness in the federal selection guidelines and its implications for minority employment. Personnel Psychology, 32, 551-562.

MulLEN, B. (1984). SELECT1 and SELECT2: BASIC computer programs for selection data analysis in industrial/organizational psychology classes. Behavior Research Methods, Instruments, \& Computers, 16, 473-474.

MuLLEN, B. (1985). SELECT3: A BASIC program for determining the consequences of using a selection procedure. Behavior Research Methods, Instruments, \& Computers, 17, 579.

(Manuscript accepted for publication January 30, 1987.) 